\title{
THE EFFECT OF STEP-SIZE ON THE NUMERICAL INTEGRATION OF SATELLITE ORBITS
}

\author{
K.G. HADJIFOTINOU \\ Department of Mathematics, Faculty of Science, Aristotle \\ University of Thessaloniki, 54006 Thessaloniki, Greece \\ AND \\ D. HARPER \\ Astronomy Unit, School of Mathematical Sciences, \\ Queen Mary and Westfield College, Mile End Road, \\ London E1 4NS, UK
}

\section{Introduction}

This work is a continuation of our study of the efficiency of two well-known methods for the numerical integration of the equations of motion of planetary satellites together with the variational equations of the system. The methods are the $10^{\text {th }}$-order Gauss-Jackson backward-difference method described in [4, 6] and the Runge-Kutta-Nyström RKN12(10)17M [1].

In our recent work [3] we determined the step-size which achieves the best combination of speed and accuracy for the Gauss-Jackson method. The Runge-Kutta-Nyström method incorporates adaptive step-size control; for this method, we investigated the optimum strategy for local error control. In both cases, we used the two-body Saturn-Mimas system and the corresponding variational equations as our test problem.

Here we present further analysis of the above results and generalise our study to more complicated systems.

\section{Study of the Gauss-Jackson method}

In [3] we presented some approximate bounds for the behaviour of the partial derivatives of the coordinates with respect to the satellite's initial conditions in the two-body case. These results are confirmed by use of Floquet 
theory [2] which predicts linear growth in the amplitude of all the partial derivatives. However, we have shown that if the step-size of the GaussJackson method is slightly increased, the system becomes unstable. This is in accordance with the findings of Milani \& Nobili [7] for the instability of multistep methods.

It is necessary to emphasise that the instability is observed only in the partial derivatives and not in the satellite's coordinates, since the error in the energy integral at the end of the integration remains small. Furthermore, we have shown that the introduction of the corrector cycle into the integration of the partial derivatives eliminates the instability and allows the use of larger step-sizes.

Investigation of the relationship between step-size of the Gauss-Jackson predictor-corrector method and the accumulation of error in the energy integral shows that it is not possible to use a step-size much greater than $T / 76$.

\section{Study of the RKN12(10)17M method}

For comparison with the Gauss-Jackson predictor-corrector method, we also considered the accumulation of the relative error in the energy integral with the mean step-size used by the RKN12(10)17M method when applied to the same system of equations. It was found that an acceptable relative error can be achieved with step-sizes as large as $T / 30$.

\section{Generalisation of results}

The above experiments have been repeated using a more generalised force model which includes the oblateness of Saturn and the mutual perturbations of Mimas and Tethys. Two-body formulae for the partial derivatives cannot be applied to such a system, but the behaviour of the partial derivatives can still be predicted by Floquet theory when the orbits are at least quasiperiodic [5]. The stability of the two numerical integration methods was found to be the same as in the two-body case.

\section{References}

Dormand J.R., El-Mikkawy M.E.A. and Prince P.J., 1987, IMA J. Num. Analysis 7, 423 Hadjidemetriou J.D., 1988, Periodic Orbits and Stability, ERASMUS ICP-88-0016-GR, Thessaloniki

Hadjifotinou K.G. and Harper D., 1995, Astron. Astrophys. 303, 940

Herrick S., 1972, Astrodynamics, Vol. 2, ed. Van Nostrand Reinhold

Jorba A., Ramirez-Ros R. and Villanueva J. (Preprint from mp_arc@math.utexas.edu \# 95-14.)

Merson R.H., 1974, RAE TR 74184

Milani A. and Nobili A.M., 1988, Celest. Mechan. 43, 1 